\title{
Ovale malaria: a case report from the Republic of Yemen
}

M.T. Al-Maktari' and H.K. Bassiouny ${ }^{2}$

\section{Introduction}

As a part of a cross-sectional research programme, a malariometric survey was conducted on an indigenous population in randomly selected centres of the Al-Hodiedah Governorate from January to December 1998. A total of 4000 thick and thin blood smears were prepared from the peripheral blood by finger prick using disposable lancets. The essential features for the processing of the slides were the use of Giemsa stain, and examination under standard conditions, i.e. 100 microscopic fields using an optical system of $\times 100$ objective and $a \times 7$ eyepiece.

A single case of Plasmodium ovale infection was detected in the Beni-Hussan village, in the Bajil district. The district lies ahout $70 \mathrm{~km}$ north of Al-Hodeidah city on the highway to Sana'a and is located between two wadis; Surdud in the north and Siham in the south.

\section{Case report}

A 26-year-old Yemeni farmer presented to a private clinic in the Bajil district on 14 July 1998 , with a history of fever and chills associated with pain all over the body and vomiting. A blood smear was taken and found negative for malaria parasites. $\mathrm{He}$ was diagnosed as having a urinary tract infection and treated accordingly, but he did not improve.

The research team took a second blood smear on 23 July 1998 . At that time the patient complained of experiencing headache, intermittent fever over a period of 10 days, loss of appetite, cough and vomiting. He had never been out of the country and he had only travelled within the Al-Hodeidah Governorate. He had no history of receiving a blond transfusion nor parenteral use of drugs. The blood film was examined on the same day by one of the authors and the patient was diagnosed as having ovale malaria. After initial diagnosis, the patient was treated with chloroquine tablets $(25 \mathrm{mg} / \mathrm{kg}$ body weight of chloroquine base divided over a period of 3 days) and primaquine tablets (15 mg base daily for 14 days). A follow-up blood smear was taken 15 days after treatment which revealed no malaria parasites.

For further confirmation, the slide was re-examined by the second author and he confirmed microscopically that this was a case of ovale malaria. The diagnosis was based upon the following: about $10 \%$ of the infected erythrocytes were distorted and

'Medical Parasitology and Entomology Department, Faculty of Medicine and Health Sciences, Sana'a University, Sand'a, Fepulilk of Yernen.

2Tropical Health Department, High Institute of Public Health, Alexandria University, Alexandria, Egypt. Received: 27/06/99; accepted: 01/07/99 
oval in shape, some had fimbria either on one or both sides, and there was irregularsized Schüffner's (James') stippling on most infected erythrocytes. Ihese characteristics confirmed that the erythrocytes were infected with $P$. ovale [1]. The growing trophozoites were compact, not amoeboid which differs from the corresponding stage of $P$. vivax and suggests less active movement. The schizonts were few, and mature forms usually had eight merozoites in a loose cluster. These features further confirmed the infection as being due to $P$. ovale [2].

Figures 1-4 show the parasites on thin Giemsa-stained blood films from the patient with their characteristic features: 1) ring stage, 2) trophozoite in a fimbriated erythrocyte, 3) male gametocyte, and 4) mature schizont with 11 merozoites in a fimbriated erythrocyte.

\section{Discussion}

The Republic of Yemen has been considered free of ovale malaria as there have been no reported indigenous cases since 1993 when two cases of $P$. ovale were recorded for the first time by the Sana'a laboratory of the Malaria Control Programme in March. Both came from the Ramah district in the Sana'a Governorate [3]. Recently however, we found an indigenous case of

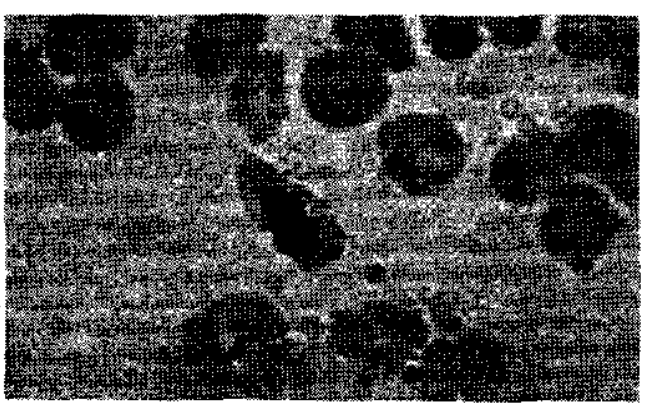

Figure 2 Trophozolte parasite In a fimbriated erythrocyte

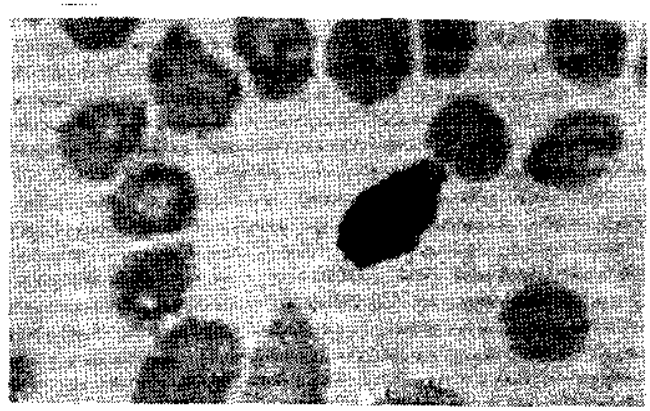

Figure 4 Mature schizont parasite with 11 morozoltee in a fimbrieted orythroeyte

Figure 3 Male gametocyte parasite 
ovale malaria in a young man who had nover been abroad.

$P$. ovale is often misdiagnosed as $P$. vivax, since proper differentiation requires extensive experience and expertise. Most microscopists find it difficult at times to differentiate between the two, particularly when the diagnosis is influenced by the assumption that ovale malaria is mostly confined to Africa. We think that this case is worth reporting as it was found in a Yemeni patient for the second time in the Republic of Yemen. It is not certain whether the present case is due to a resurgence of indigenous ovale malaria, or a secondary infection from ovale malaria which has been introduced to the country as many African people come to work and live in the Republic of Yemen, mostly as refugees from Somalia, Ethiopia and Eritrea. According to a 1998 report by the Office of the United $\mathrm{Na}$ - tions High Commissioner for Refugees (UNHCR), 91000 individuals came to the Republic of Yemen because of political instability and civil wars in these ncighbouring countries [4]. Whichever the source of the infection, the presence of autochthonous ovale malaria in the country should require public health workers to consider the existence of this particular Plasmodium species when surveying for malaria in the country in the future.

Microscopists working in malaria laboratories and private clinics in the Republic of Yemen should periodically be trained in malaria microscopy by the Ministry of Health. The purpose of this would be to emphasize the morphological details of malaria parasites, since examination of blood film remains the most reliable test for the diagnosis of malaria species.

\section{References}

1. Matsumoto Y, Matsuda S, Yoshida Y. UItrastructure of human erythrocytes infected with Plasmodium ovale. American joumal of tropical medicine and hygiene, 1986, 35:697-703.

2. Matsumoto $Y$, Matsuda S, Yoshida Y. Ultrastructure of erythyrocytic stages of Plasmodium ovale in humans. American journal of tropical medicine and hygiene, 1986, 35:689-96.
3. Annual descriptive report of the Malaria Control Programme. Sana'a, Republlc of Yemen, Ministry of Health, 1993.

4. Facts and numbers. Sana'a, Republic of Yemen, United Nations High Commissioner for Refugees, 1998. 